\title{
Incidência de pinta preta e características físico-químicas de goiabas submetidas a tratamentos pós-colheita
}

\author{
Ivan Herman Fischer ${ }^{*}$, Maria Cecília de Arruda Palharini', Ana Paula dos Santos', \\ Antônio Fernandes Nogueira Júnior ${ }^{2}$, Marise Cagnin Martins Parisi ${ }^{3}$ \\ 'Agência Paulista de Tecnologia dos Agronegócios, Bauru, SP, Brasil \\ ${ }^{2}$ Universidade de São Paulo, Piracicaba, SP, Brasil \\ Agência Paulista de Tecnologia dos Agronegócios, Piracicaba, SP, Brasi \\ *Autor correspondente, e-mail: infische@apta.sp.gov.br
}

\begin{abstract}
Resumo
A goiaba apresenta elevada perecibilidade decorrente do seu intenso metabolismo e da ocorrência de patologias no período pós-colheita. Em virturde da carência de tecnologias de conservação de goiabas, objetivou-se avaliar o efeito de produtos alternativos [fosfito de potássio, cloreto de cálcio, fécula de mandioca, 1-metilciclopropeno (1-MCP), etanol seguido de dicloro s. triazinatriona sódica dihidratada (etanol+cloro)] e de hidrotermia, isoladamente e em associação, no controle da pinta preta e nas características físico-químicas de goiabas 'Pedro Sato'. Os tratamentos foram avaliados em goiabas infectadas em condições de campo, em três estádios de maturação, armazenadas a $22-25^{\circ} \mathrm{C}$ e $80-85 \%$ de UR, por 8 dias. A associação de tratamento etanol+cloro/fécula de mandioca reduziu a incidência de pinta preta nos três estádios de maturação de goiabas, permitindo um maior período de conservação dos frutos, com um atraso de dois a quatro dias no aparecimento dos sintomas da doença. A manutenção da qualidade físico-química das goiabas com as associações de tratamentos foi evidenciada pelo atraso na mudança de coloração da casca e menor redução na firmeza da polpa. Observou-se correlação entre os parâmetros incidência de pinta preta e cor da casca, ou seja, frutos colhidos em estádios mais avançados de maturação apresentaram maior incidência da doença. A maior eficiência da associação etanol+cloro/fécula no controle da pinta preta relacionou-se diretamente com o atraso no amadurecimemento dos frutos, evidenciado pelos atributos cor da casca e firmeza da polpa.
\end{abstract}

Palavras-chave: Guignardia, Psidium guajava, manejo, qualidade pós-colheita

Black spot incidence and physic-chemical properties of guavas submitted to postharvest treatments

\begin{abstract}
The guava fruit is very as high perishable due to its intense metabolism and the occurrence of postharvest diseases. Due to the lack conservation technologies for this fruit, this study aimed to evaluate the effect of alternative products [potassium phosphite, calcium chloride, cassava starch edible coat, 1-methylcyclopropene (1-MCP), ethanol followed by dichloro s. triazinatriona sodium (ethanol+chlorine)] and hot water treatments, singly or in combination, in black spot control and the physic-chemical characteristics of 'Pedro Sato' guavas. The treatments were evaluated in naturally infected guavas at three maturity stages and stored at $22-25^{\circ} \mathrm{C}$ and $80-85 \% \mathrm{RH}$ for 8 days. The treatment association of ethanol+chlorine and cassava starch reduced the incidence of black spot in three maturity stages of guavas, allowing the increase of fruit's shelf life, with a delay of two to four days in the onset of black spot symptoms. The maintenance of the physical and chemical quality characteristics of guavas with association of treatments were evidenced by the delay in skin color change, and a smaller decrease in firmness. Correlation occurred between the black spot incidence and the skin color parameter, ie, fruits harvested at later maturity stages presented higher incidence of the disease. The greater efficiency of ethanol+chlorine/starch association in the control of black spot was directly related to the ripening delay in fruits, evidenced by the skin color and firmness attributes.
\end{abstract}

Keywords: Guignardia, Psidium guajava, management, postharvest quality 


\section{Introdução}

A goiabeira (Psidium guajava L.) é uma planta pertencente à família Myrtaceae, nativa da América Tropical (Risterucci et al., 2005). No Brasil, a cultura concentra-se nas regiões Nordeste e Sudeste, sendo destaque os Estados de São Paulo e Pernambuco. A cultivar Pedro Sato é uma das mais cultivadas goiabas de polpa vermelha para consumo in natura (Hojo et al., 2007), apresentando frutos grandes, de casca rugosa, formato oblongo, polpa rosada, espessa e com poucas sementes.

A expansão da comercializaçãoin natura está ligada à qualidade dos frutos e ao aumento da sua vida pós-colheita. Contudo, em função do alto metabolismo do fruto, apresenta elevada perecebilidade. O estádio de maturação em que é o fruto é colhido determina a qualidade a ser oferecida ao consumidor, devendo ser colhido após a maturidade fisiológica, como forma de preservar suas qualidades organolépticas, preferencialmente no estádio 2 (Cavaline et al., 2006), quando apresentam boa resistência ao transporte. Frutos colhidos muito maduros entram rapidamente em senescência, devido, principalmente, as doenças (Azzolini et al.; 2004). Segundo Azzolini et al. (2004) frutos do estádio 3 apresentaram melhor sabor e textura em relação à frutos colhidos no estádio 1 e 2, no entanto os frutos de todos os estádios foram aceitos sensorialmente.

As doenças pós-colheita comprometem a qualidade e a produção dos frutos. As doenças quiescentes, cuja infecção ocorre desde o início da formação do fruto até sua manipulação na pós-colheita, são as mais importantes em goiaba, como é o caso da pinta preta, causada por Guignardia psidii e da antracnose, causada por Colletotrichum spp. (Martins et al., 2007; Fischer et al., 2011, Fischer et al., 2012). A incidência média de pinta preta e de antracnose atingiu valores superiores a $30 \%$ em goiabas comercializadas na Companhia de Entrepostos e Armazéns Gerais de São Paulo (CEAGESP) e armazenadas por dez dias a $25^{\circ} \mathrm{C}$ (Soares-Colletti et al., 2014).

A falta de tecnologias de conservação limita o período de comercialização, tendo por consequência a redução do número de mercados consumidores. Devido à crescente restrição ao uso de fungicidas em pós-colheita, alternativas visando uma maior conservação estão sendo estudadas, algumas com alguma eficiência em goiabas, como hidrotermia (Ferraz, 2010), inibidor da ação do etileno (Basseto et al., 2005; Linhares et al., 2007), cálcio (Linhares et al., 2007; Ferraz, 2010), fosfitos (Ferraz, 2010), álcool (Ponzo, 2009) e fécula de mandioca (Cerqueira, 2007, Vila et al., 2007), responsáveis por atuar na fisiologia dos frutos, retardando seu amadurecimento e aumentando sua resistência à patógenos. A eficiência no controle da doença pode ser aumentada pela combinação de tratamentos, como observado para a antracnose, com hidrotermia, cloreto de cálcio e fosfito de potássio (Ferraz, 2010).

Objetivou-se no presente trabalho avaliar o efeito de produtos alternativos e da hidrotermia, isoladamente e em associação, no controle da pinta preta e nas características físico-químicas de goiabas em três estádios de maturação.

\section{Material e Métodos}

Goiabas 'Pedro Sato' provenientes de pomar comercial com histórico de ocorrência de pinta preta, localizado no município de Itajú/ SP foram utilizadas, advindas de lotes uniformes, com frutos de aproximadamente 300 gramas, sem defeitos e com o estádio de maturação caracterizado com base na cor da casca (estádio 1: cor da casca verde-escura, ângulo de cor ( $\mathrm{h}$ ) entre 120 e 117; estádio 2: cor da casca verde-clara, oh entre 116 e 113 e estádio 3: cor da casca verde-amarela, oh entre 112 e 108), de acordo com Azzolini et al. (2004), com o auxílio de colorímetro Minolta, modelo CR-400.

Os experimentos foram divididos em três fases. Na primeira fase, goiabas no estádio 2 de maturação foram submetidas a sete tratamentos por imersão (tempo de imersão): 1) água destilada (10 minutos- tratamento controle); 2) $20 \mathrm{~g} \mathrm{~L}^{-1}$ de cloreto de cálcio (20 minutos); 3) fosfito $\mathrm{K}\left(40 \% \mathrm{P}_{2} \mathrm{O}_{5}+20 \% \mathrm{~K}_{2} \mathrm{O}\right.$ - 'Fitofós K plus' 1,5 $\left.\mathrm{mL} \mathrm{L} \mathrm{L}^{-1}\right)$ (20 minutos) (Ferraz, 2010); 4) hidrotermia a $50^{\circ} \mathrm{C}$ (10 minutos) (Ponzo, 2009); 5) $900 \mathrm{~nL} \mathrm{~L}-1$ de 1-metilciclopropeno (1-MCP) durante três horas de exposição ao gás (Basseto et al., 2005); 6) $30 \mathrm{~g} \mathrm{~L}^{-1}$ de fécula de mandioca (10 segundos) 
Fischer et al. (2016) / Incidência de pinta preta e características...

(Cerqueira, 2007); 7) etanol 50\% (5 minutos). seguido de dicloro s. triazinatriona sódica dihidratada $0,2 \mathrm{~g} \mathrm{~L}^{-1}$ de cloro ativo (5 minutos). Após secagem em ambiente de laboratório, os frutos foram individualizados em bandejas plásticas $(28 \times 48 \mathrm{~cm})$, revestidas por bandejas PET separadoras e submetidos a armazenamento por oito dias a $22^{\circ} \mathrm{C}$ e $80-85 \%$ de UR, em câmara fria. A incidência de pinta preta decorrente de infecção natural no campo foi avaliada visualmente a cada dois dias, identificando a doença com base na sintomatologia e na observação das estruturas do agente causal sob microscópio óptico.

Em uma segunda fase do experimento, os quatro produtos que proporcionaram redução na incidência de pinta preta foram selecionados e avaliados em associação, aplicados de forma sequencial, novamente em frutos no estádio 2 de maturação. O armazenamento dos frutos e a avaliação da pinta preta foram realizados conforme descritos na primeira fase, exceto pela temperatura de armazenamento. Optou-se por elevar a temperatura de 22 para $25^{\circ} \mathrm{C}$, de modo que a eficiência dos tratamentos fosse avaliada na condição ideal de desenvolvimento da pinta preta. Segundo Escanferla et al. (2009), temperaturas em torno de $25^{\circ} \mathrm{C}$ são mais favoráveis à infecção de goiabas por $\mathrm{G}$. psidii.

Três combinações de tratamentos que proporcionaram redução na incidência de pinta preta foram selecionadas e avaliadas novamente em frutos colhidos em três estádios de maturação (1, 2 e 3) e armazenados por oito dias a $22^{\circ} \mathrm{C}$ e $80-85 \%$ de UR, em câmara fria.

O delineamento experimental utilizado foi o inteiramente casualizado, em fatorial na terceira fase do experimento (quatro tratamentos $x$ três estádios de maturação), adotando-se três repetições de 5-10 frutos por parcela para cada tratamento. Os experimentos foram repetidos duas vezes. A incidência da pinta preta foi comparada entre os tratamentos e os estádios de maturação, durante o armazenamento, por meio de análise de variância e as médias comparadas pelo teste não paramétrico de comparação de múltiplas proporções $(p<0,05)$ (Zar, 1999), pois os dados não apresentaram distribuição normal. Sempre que significativo, as proporções foram comparadas após as seguintes transformações: $\left[\mathrm{Pi}=\mathrm{X} /(\mathrm{n}+1), \mathrm{Pi}^{\prime}=(\mathrm{X}+1) /(\mathrm{n}+1)\right.$, e $\mathrm{Pi}^{\prime \prime}=1 / 2$ (asen $\mathrm{Pi}+$ asen $\left./ \mathrm{Pi}^{\prime}\right)$ ), onde: $\mathrm{X}=$ número de frutos totais de cada proporção; $n=$ número de frutos doentes de cada proporção e Pi"'= Proporção transformada, de acordo com Zar (1999).

Em adição às avaliações de pinta preta, na terceira fase dos experimentos foi feita a caracterização físico-química dos frutos submetidos aos diferentes tratamentos após a colheita, considerado dia zero, e após oito dias de armazenamento nas mesmas condições descritas para os frutos avaliados para pinta preta. As características físico-químicas avaliadas foram: a) cor da casca, determinada usando-se colorímetro Minolta, modelo CR-400; b) firmeza da polpa, determinada com penetrômetro digital, ponteira $8 \mathrm{~mm}$, tomando-se duas leituras na região equatorial de cada fruto; $c$ ) perda de massa fresca (g), determinada em balança de precisão com duas casas decimais, d) teor de sólidos solúveis, determinado em refratômetro digital Atago, modelo PAL-1; e) teor de acidez titulável, determinado por titulação com $\mathrm{NaOH}$ e f) ratio, obtido por meio da razão entre sólidos solúveis e acidez tiulável. $O$ delineamento experimental foi o inteiramente casualizado em esquema fatorial (quatro tratamentos $x$ três estádios de maturação), com três repetições de quatro frutos para cada tratamento. Cada parâmetro físico-químico avaliado foi comparado entre os tratamentos e estádios de maturação, por meio de análise de variância e as médias comparadas pelo teste de Tukey $(p<0,05)$. As variáveis físico-químicas foram correlacionadas com a incidência de pinta preta utilizando-se a correlação de Spearman $(p<0,05)$ com auxilio do software Plotit.

\section{Resultados}

Controle alternativo da pinta preta da goiaba Na primeira fase da pesquisa em que foram avaliados sete tratamentos, constatouse redução significativa da pinta preta com o tratamento fécula de mandioca a partir do sexto dia de armazenamento e com 1-MCP a partir do oitavo dia (Tabela 1). Embora os demais tratamentos tenham sido ineficientes no controle 
da pinta preta, foram selecionados, em adição a fécula e ao 1-MCP, os tratamentos hidrotermia e etanol+cloro, para serem avaliados em associação de dois, aplicados de forma sequencial, pois estes apresentaram redução da antracnose (dados não apresentados).

Tabela 1. Efeito de tratamentos alternativos pós-colheita no controle da pinta preta da goiaba 'Pedro Sato', durante oito dias de armazenamento a $22^{\circ} \mathrm{Ce} 80-85 \%$ de UR

\begin{tabular}{ccccc}
\hline Tratamentos & \multicolumn{4}{c}{ Incidência (\%) da pinta preta' } \\
\cline { 2 - 5 } & 2 & -dias de armazenamento- & 8 \\
\hline Fécula de mandioca & $2,4 \mathrm{a}^{2}$ & $2,4 \mathrm{a}$ & $9,5 \mathrm{c}$ & $19,0 \mathrm{c}$ \\
1-MCP & $0,0 \mathrm{a}$ & $14,3 \mathrm{a}$ & $23,8 \mathrm{bc}$ & $26,2 \mathrm{c}$ \\
Fosfito de potássio & $2,4 \mathrm{a}$ & $19,0 \mathrm{a}$ & $38,1 \mathrm{ab}$ & $50,0 \mathrm{bc}$ \\
Cloreto de cálcio & $0,0 \mathrm{a}$ & $14,3 \mathrm{a}$ & $45,2 \mathrm{ab}$ & $64,3 \mathrm{ab}$ \\
Hidrotermia & $0,0 \mathrm{a}$ & $4,8 \mathrm{a}$ & $64,3 \mathrm{a}$ & $85,7 \mathrm{a}$ \\
Etanol+cloro & $0,0 \mathrm{a}$ & $11,9 \mathrm{a}$ & $40,5 \mathrm{ab}$ & $66,7 \mathrm{ab}$ \\
Controle & $2,4 \mathrm{a}$ & $19,0 \mathrm{a}$ & $45,2 \mathrm{ab}$ & $61,9 \mathrm{ab}$ \\
\hline 'Média (\%) de três experimentos, com 15 frutos para cada tratamento. ${ }^{2}$ Médias seguidas pela mesma letra na coluna não diferem estatisticamente entre si \\
em nivel de 5\% pelo teste não paramétrico de comparação de mútliplas proporcōes
\end{tabular}

As três associações de tratamentos com fécula de mandioca foram eficientes em atrasar em pelo menos dois dias o aparecimento dos sintomas de pinta preta, com destaque para etanol+cloro/fécula e hidrotermia/fécula já a partir do quarto dia de armazenamento (Tabela 2). Etanol+cloro/termoterapia propiciou um aumento na incidência em relação ao controle, a partir do sexto dia.

Tabela 2. Efeito de tratamentos alternativos pós-colheita no controle da pinta preta da goiaba 'Pedro Sato', durante oito dias de armazenamento a $25^{\circ} \mathrm{C}$ e $80-85 \%$ de UR

\begin{tabular}{ccccc}
\hline Tratamentos & \multicolumn{4}{c}{ Incidência (\%) da pinta preta' } \\
\cline { 2 - 5 } & 2 & -dias de armazenamento- & 8 \\
\hline Controle & $3,3 \mathrm{a}^{2}$ & $28,9 \mathrm{bc}$ & $75,6 \mathrm{~cd}$ & $92,2 \mathrm{de}$ \\
Etanol+cloro/hidrotermia & $0,0 \mathrm{a}$ & $14,4 \mathrm{abc}$ & $97,8 \mathrm{e}$ & $100,0 \mathrm{f}$ \\
Etanol+cloro/fécula de mandioca & $1,1 \mathrm{a}$ & $6,7 \mathrm{a}$ & $24,4 \mathrm{a}$ & $41,1 \mathrm{a}$ \\
Etanol+cloro/1-MCP & $1,1 \mathrm{a}$ & $31,1 \mathrm{c}$ & $72,2 \mathrm{bc}$ & $80,0 \mathrm{~cd}$ \\
Hidrotermia/fécula de mandioca & $2,2 \mathrm{a}$ & $7,8 \mathrm{a}$ & $51,1 \mathrm{~b}$ & $67,8 \mathrm{bc}$ \\
Hidrotermia /1-MCP & $0,0 \mathrm{a}$ & $28,1 \mathrm{bc}$ & $94,4 \mathrm{de}$ & $96,6 \mathrm{ef}$ \\
1-MCP/fécula de mandioca & $2,2 \mathrm{a}$ & $12,2 \mathrm{ab}$ & $50,0 \mathrm{~b}$ & $57,8 \mathrm{ab}$ \\
\hline Média (\%) de três experimentos, com 30 frutos para cada tratamento. ${ }^{2}$ Médias seguidas pela mesma letra na coluna não diferem estatisticamente entre siem nivel
\end{tabular}

de $5 \%$ pelo teste não paramétrico de comparação de múltiplas proporções.

A ocorrência da pinta preta foi maior no tratamento controle na segunda fase dos experimentos (Tabela 2) em relação à primeira fase (Tabela 1). Analisando-se a eficiência dos tratamentos isoladamente e em associação em relação ao respectivo tratamento controle, praticamente não ocorreu diferença na média dos tratamentos nas fases 1 e 2, ao final do armazenamento, com redução da incidência de 20,2 e 19,9\%, respectivamente. Para a terceira fase dos experimentos, optou-se por selecionar o tratamento etanol+cloro/1-MCP ao invés do tratamento 1-MCP/fécula, pois o primeiro foi mais eficiente no controle da antracnose (dados não apresentados).

Das três associações de tratamentos avaliadas na terceira fase (etanol+cloro/fécula de mandioca, hidrotermia/fécula de mandioca e etanol+cloro/1-MCP), etanol+cloro/fécula foi o único tratamento que controlou a doença nos três estádios de maturação, atrasando em pelo menos 4 dias a ocorrência da doença em relação ao controle nos estádios 1 e 2 de maturação (Tabela 3). O tratamento hidrotermia/ fécula foi eficiente em controlar a doença nos estádios 2 e 3 de maturação, entretanto foi inferior ao etanol+cloro/fécula no estádio 2 de maturação. Já o tratamento etanol+cloro/1MCP foi ineficiente em controlar a pinta preta. O progresso da doença foi crescente com o estádio de maturação a partir do quarto dia de armazenamento, sendo significativo em todos os 
Fischer et al. (2016) / Incidência de pinta preta e características...

tratamentos a partir do sexto dia. Estes resultados

etanol+cloro/fécula e hidrotermia/fécula e são condizentes aos observados na segunda ineficácia de etanol+cloro/1-MCP. fase da pesquisa, com eficácia das associações

Tabela 3. Efeito de tratamentos alternativos pós-colheita e estádios de maturação na incidência (\%) de pinta preta em goiaba 'Pedro Sato', durante oito dias de armazenamento a $22^{\circ} \mathrm{C}$ e $80-85 \%$ de UR.

\begin{tabular}{|c|c|c|c|}
\hline \multirow[t]{2}{*}{ Tratamentos } & \multicolumn{3}{|c|}{ Estádio de maturação' } \\
\hline & 1 & 2 & 3 \\
\hline & \multicolumn{3}{|c|}{-2 dias de armazenamento- } \\
\hline Controle & $0,0 \mathrm{aA}$ & $0,0 \mathrm{aA}$ & $0,0 \mathrm{aA}$ \\
\hline Etanol+cloro/fécula de mandioca & $0,0 \mathrm{aA}$ & $0,0 \mathrm{aA}$ & $0,0 \mathrm{aA}$ \\
\hline Etanol+cloro /1-MCP & $0,0 \mathrm{aA}$ & $0,0 \mathrm{aA}$ & $0,0 \mathrm{aA}$ \\
\hline \multirow[t]{2}{*}{ Hidrotermia/fécula de mandioca } & $0,0 \mathrm{aA}$ & $0,0 \mathrm{aA}$ & $0,0 \mathrm{aA}$ \\
\hline & \multicolumn{3}{|c|}{-4 dias de armazenamento- } \\
\hline Controle & $2,2 \mathrm{aA}$ & $15,6 \mathrm{abB}$ & $35,6 \mathrm{bB}$ \\
\hline Etanol+cloro/fécula de mandioca & $0,0 a \mathrm{~A}$ & $0,0 a \mathrm{~A}$ & $2,2 \mathrm{aA}$ \\
\hline Ełanol+cloro /1-MCP & $0,0 \mathrm{aA}$ & $8,9 \mathrm{abAB}$ & $26,7 \mathrm{bB}$ \\
\hline \multirow[t]{2}{*}{ Hidrotermia/fécula de mandioca } & $0,0 \mathrm{aA}$ & $0,0 \mathrm{aA}$ & $2,2 \mathrm{aA}$ \\
\hline & \multicolumn{3}{|c|}{-6 dias de armazenamento- } \\
\hline Controle & $15,6 \mathrm{aB}$ & $57,8 \mathrm{bc}$ & $86,7 \mathrm{CB}$ \\
\hline Etanol+cloro/fécula de mandioca & $0,0 \mathrm{aA}$ & $2,2 \mathrm{aA}$ & $46,7 \mathrm{bA}$ \\
\hline Ełanol+cloro / 1-MCP & $46,7 \mathrm{aC}$ & $71,1 \mathrm{abc}$ & $91,1 \mathrm{bB}$ \\
\hline \multirow[t]{2}{*}{ Hidrotermia/fécula de mandioca } & $6,7 \mathrm{aAB}$ & $24,4 \mathrm{aB}$ & $51,1 \mathrm{bA}$ \\
\hline & \multicolumn{3}{|c|}{-8 dias de armazenamento- } \\
\hline Controle & $51,1 \mathrm{aBC}$ & $86,7 \mathrm{bC}$ & $100,0 \mathrm{cC}$ \\
\hline Etanol+cloro/fécula de mandioca & $2,2 \mathrm{aA}$ & 15,6 aA & $80,0 \mathrm{bA}$ \\
\hline Etanol+cloro /1-MCP & $60,0 \mathrm{aC}$ & 86,7 bC & $97,8 \mathrm{bBC}$ \\
\hline Hidrotermia/fécula de mandioca & $28,9 \mathrm{aB}$ & $60,0 \mathrm{bB}$ & $84,4 \mathrm{CAB}$ \\
\hline
\end{tabular}

Caracterização físico-química dos frutos submetidos aos tratamentos alternativos

Os frutos colhidos em três estádios de maturação com base na cor da casca, apresentaram, em adição a característica cor da casca, diferenças na firmeza da polpa, acidez titulável e ratio (Tabela 4), não havendo diferenças em sólidos solúveis em função do estádio de maturação.

A perda de massa dos frutos foi semelhante entre os tratamentos $(p<0,05)$ ao final do armazenamento (Tabela 5), ou seja, os tratamentos não foram efetivos em retardar a perda de massa nos três estádios de maturação. Em relação à cor da casca as três associações de tratamentos, nos três estádios de maturação da fruta apresentaram maior ângulo de cor, diferindo do controle. Os frutos submetidos as associações de tratamentos apresentaram maior firmeza em relação ao tratamento controle, exceto o tratamento etanol+cloro/fécula que se assemelhou ao controle no estádio 3 de maturação.

Tabela 4. Características físico-químicas de goiabas 'Pedro Sato' colhidas em três estádios de maturação com base da cor da casca.

\begin{tabular}{cccc}
\hline Variáveis & \multicolumn{3}{c}{ Estádio de maturação } \\
\cline { 2 - 4 } & 1 & 2 & 3 \\
\hline Cor casca ( ${ }^{\circ}$ hue) & $117,67 \mathrm{~A}$ & $114,78 \mathrm{~B}$ & $109,96 \mathrm{C}$ \\
Firmeza da polpa (N) & $108,30 \mathrm{~A}$ & $65,90 \mathrm{~B}$ & $44,70 \mathrm{C}$ \\
Sólidos solúveis ( $\left.{ }^{\circ} \mathrm{Brix}\right)$ & $9,9 \mathrm{~A}$ & $9,4 \mathrm{~A}$ & $9,5 \mathrm{~A}$ \\
Acidez tituável(\% ác. cítrico) & $0,63 \mathrm{~A}$ & $0,57 \mathrm{~B}$ & $0,50 \mathrm{C}$ \\
Ratio & $15,77 \mathrm{C}$ & $16,54 \mathrm{~B}$ & $19,13 \mathrm{~A}$ \\
\hline Média (\%) de três experimentos, com 12 frutos para cada tratamento. ${ }^{2}$ Médias seguidas pela mesma letra na linha não diferem estatisticamente entre si \\
em nivel de 5\% pelo teste de Tukey.
\end{tabular}

Observou-se maior perda de massa dos frutos ao final do armazenamento conforme

avanço do estádio de maturação na colheita, assim como as diferenças na cor da casca e na 
firmeza da polpa, constatadas entre os estádios final do armazenamento (Tabela 5). de maturação na colheita, permaneceram ao

Tabela 5. Efeito de tratamentos alternativos em pós-colheita e estádios de maturação nas características físicas de goiabas 'Pedro Sato', aos 8 dias de armazenamento a $22^{\circ} \mathrm{C}$ e $80-85 \%$ de UR'

\begin{tabular}{cccc}
\hline Tratamentos & \multicolumn{3}{c}{ Estádio de maturação } \\
\cline { 2 - 4 } & 1 & 2 & 3 \\
\hline Controle & $4,24 \mathrm{aC}$ & Perda de massa (\%) \\
Etanol+cloro/fécula & $3,72 \mathrm{aC}$ & $5,30 \mathrm{aB}$ & $7,19 \mathrm{aA}$ \\
Etanol+cloro/1-MCP & $4,00 \mathrm{aC}$ & $4,31 \mathrm{aB}$ & $7,26 \mathrm{aA}$ \\
Hidrotermia/fécula & $3,78 \mathrm{aC}$ & $4,88 \mathrm{aB}$ & $7,47 \mathrm{aA}$ \\
& & $4,38 \mathrm{aB}$ & $7,28 \mathrm{aA}$ \\
Controle & $93,61 \mathrm{bA}$ & Cor casca ( ${ }^{\circ}$ hue) & $89,06 \mathrm{bB}$ \\
Etanol+cloro/fécula & $113,86 \mathrm{aA}$ & $89,33 \mathrm{bB}$ & $101,72 \mathrm{aC}$ \\
Etanol+cloro/1-MCP & $113,75 \mathrm{aA}$ & $109,81 \mathrm{aB}$ & $101,76 \mathrm{aC}$ \\
Hidrotermia/fécula & $114,02 \mathrm{aA}$ & $109,78 \mathrm{aB}$ & $103,73 \mathrm{aC}$ \\
& & $109,09 \mathrm{aB}$ & \\
Controle & $18,41 \mathrm{bA}$ & Firmeza da polpa (N) & $8,21 \mathrm{bB}$ \\
Etanol+cloro/fécula & $50,71 \mathrm{aA}$ & $12,25 \mathrm{bAB}$ & $13,39 \mathrm{abC}$ \\
Etanol+cloro/1-MCP & $46,64 \mathrm{aA}$ & $31,12 \mathrm{aB}$ & $17,97 \mathrm{aC}$ \\
Hidrotermia/fécula & $43,58 \mathrm{aA}$ & $29,39 \mathrm{aB}$ & $16,06 \mathrm{aB}$ \\
\hline Média (\%) de três experimentos, com 12 frutos para cada tratamento. ${ }^{2}$ Médias seguidas pela mesma letra, minúscula na coluna e maíscula na linha, não \\
diferem estatisticamente entre si em nivel de 5\% pelo teste de Tukey.
\end{tabular}

diferem estatisticamente entre si em nivel de $5 \%$ pelo teste de Tukey.

Os frutos do estádio 1 de maturação submetidos às associações de tratamentos apresentaram maior teor de sólidos solúveis em relação ao tratamento controle, enquanto que no estádio 2 de maturação os frutos tratados com etanol+cloro/fécula e etanol+cloro/1MCP apresentaram maior teor. No estádio 3 apenas os frutos tratados com etanol+cloro/1-MCP apresentaram maior teor (Tabela 6).

Observou-se que os frutos submetidos às associações de tratamentos apresentaram menor acidez titulável no estádio mais avançado de maturação. Os frutos tratados com etanol+cloro/1-MCP apresentaram maior acidez nos três estádios de maturação, diferindo do controle. O mesmo foi observado no tratamento etanol+cloro/fécula nos estádio 2 e 3 (Tabela 6).

Menores teores de acidez titulável foram observados com o avanço do estádio de maturação em todos os tratamentos, enquanto que para sólidos solúveis um menor teor foi observado nos tratamenos etanol+cloro/fécula e hidrotermia/fécula nos estádios mais avançados de maturação (Tabela 6).

O ratio foi maior nos estádios mais avançados de maturação (Tabela 6). No estádio 1 o ratio dos frutos tratados assemelhouse ao controle. Já no estádio 2, os frutos tratados com etanol+cloro/fécula e etanol+cloro /1-
MCP diferiram do controle enquanto no estádio 3 todos os tratamentos diferiram do controle, apresentando menor ratio.

Análise de correlação entre pinta preta e parâmetros físico-químicos

Observou-se correlação negativa ( $\mathrm{r}=$ -0,81) para os parâmetros incidência de pinta preta em relação ao atributo cor da casca, em goiabas amostradas em três estádios de maturação e armazenadas por oito dias a $22^{\circ} \mathrm{C}$, ou seja, frutos com maior maturação (coloração amarela mais acentuada) apresentaram maior incidência da doença. Em relação aos demais parâmetros físico-químicos avaliados não foi observada correlação significativa em relação a incidência de pinta preta, em frutos amostrados em três estádios de maturação.

Com relação à correlação entre a incidência da pinta preta em relação aos parâmetros físico-químicos em goiabas submetidas a diferentes tratamentos póscolheita, armazenadas por oito dias a $22^{\circ} \mathrm{C}$, observou-se uma correlação negativa para pinta preta e os parâmetros cor da casca e firmeza da polpa em frutos submetidos ao tratamento etanol+cloro/fécula de mandioca, nos três estádios de maturação (Tabela 7). 
Fischer et al. (2016) / Incidência de pinta preta e características...

Tabela 6. Efeito de tratamentos alternativos em pós-colheita e estádios de maturação nas características químicas de goiabas 'Pedro Sato', aos 8 dias de armazenamento a $22^{\circ} \mathrm{C}$ e $80-85 \%$ de UR'

\begin{tabular}{|c|c|c|c|}
\hline \multirow[t]{2}{*}{ Tratamentos } & \multicolumn{3}{|c|}{ Estádio de maturação } \\
\hline & 1 & 2 & 3 \\
\hline & \multicolumn{3}{|c|}{ Sólidos solúveis ('Brix) } \\
\hline Controle & $9,2 \mathrm{bA}$ & $9,3 \mathrm{bA}$ & $9,2 \mathrm{bA}$ \\
\hline Ełanol+cloro/fécula & $9,7 \mathrm{aA}$ & $9,8 a \mathrm{~A}$ & $9,0 \mathrm{bB}$ \\
\hline Etanol+cloro/1-MCP & $10,0 \mathrm{aA}$ & $9,8 \mathrm{aA}$ & 9,8 aA \\
\hline Hidrotermia/fécula & $9,7 \mathrm{aA}$ & $9,4 \mathrm{bAB}$ & $9,1 \mathrm{bB}$ \\
\hline & \multicolumn{3}{|c|}{ Acidez tituável (\% ác. cítrico) } \\
\hline Controle & $0,68 \mathrm{bA}$ & $0,53 \mathrm{cB}$ & $0,44 \mathrm{CC}$ \\
\hline Ełanol+cloro/fécula & $0,68 \mathrm{bA}$ & $0,62 \mathrm{bB}$ & $0,50 \mathrm{bc}$ \\
\hline Ełanol+cloro/1-MCP & $0,75 \mathrm{aA}$ & $0,69 \mathrm{aB}$ & $0,55 \mathrm{aC}$ \\
\hline Hidrotermia/fécula & $0,69 \mathrm{bA}$ & $\begin{array}{c}0,57 \mathrm{cB} \\
\text { Ratio }\end{array}$ & $0,52 \mathrm{abc}$ \\
\hline Controle & $13,53 \mathrm{aC}$ & $17,55 a B$ & $20,19 \mathrm{aA}$ \\
\hline Ełanol+cloro/fécula & 14,26 aC & $15,891 \mathrm{bB}$ & $18,00 \mathrm{bA}$ \\
\hline Ełanol+cloro/1-MCP & $13,33 \mathrm{aB}$ & $14,20 \mathrm{cB}$ & $17,82 \mathrm{bA}$ \\
\hline Hidrotermia/fécula & $14,06 \mathrm{aB}$ & $16,49 \mathrm{abA}$ & $17,50 \mathrm{bA}$ \\
\hline
\end{tabular}

Tabela 7. Coeficientes de correlação de Spearman (r) entre a incidência de pinta preta e parâmetros físicoquímicos em goiabas 'Pedro Sato' amostradas em três estádios de maturação, segundo a cor da casca ( $1=v e r d e$ escuro; $2=v e r d e$ calor e 3=verde-amarelo) e submetidas a diferentes tratamentos pós-colheita, armazenadas por oito dias a $22^{\circ} \mathrm{C}$

\begin{tabular}{|c|c|c|c|c|c|}
\hline \multirow{2}{*}{ Tratamentos } & \multicolumn{5}{|c|}{ Parâmetros físico-químicos } \\
\hline & Cor da casca & $\begin{array}{c}\text { Firmeza da } \\
\text { polpa }\end{array}$ & Sólidos solúveis & $\begin{array}{l}\text { Acidez } \\
\text { titulável }\end{array}$ & Ratio \\
\hline & & Estádio 1 & & & \\
\hline Etanol+cloro/fécula & $-0,91^{*}$ & $-0,88^{*}$ & 0,66 & 0,03 & 0,17 \\
\hline Etanol+cloro/1-MCP & 0,24 & 0,15 & 0,53 & $-0,11$ & 0,29 \\
\hline Hidrotermia/fécula & $-0,56$ & $\begin{array}{l}-0,09 \\
\text { Estádio } 2\end{array}$ & 0,35 & 0,31 & $-0,19$ \\
\hline Ełanol+cloro/fécula & $-0,97^{*}$ & $-0,85^{*}$ & 0,52 & 0,61 & $-0,32$ \\
\hline Etanol+cloro/1-MCP & $-0,09$ & 0,16 & 0,78 & 0,64 & $-0,08$ \\
\hline Hidrotermia/fécula & $-0,75$ & $\begin{array}{l}-0,58 \\
\text { Estádio } 3\end{array}$ & 0,28 & 0,66 & $-0,70$ \\
\hline Etanol+cloro/fécula & $-0,84^{*}$ & $-0,97^{*}$ & $-0,35$ & $-0,28$ & 0,10 \\
\hline Etanol+cloro/1-MCP & $-0,57$ & $-0,62$ & 0,01 & $-0,59$ & 0,62 \\
\hline Hidrotermia/fécula & $-0,78$ & $-0,30$ & $-0,24$ & 0,29 & $-0,50$ \\
\hline
\end{tabular}

\section{Discussão}

Os tratamentos fécula de mandioca e 1-MCP reduziram a incidência de pinta preta em goiabas 'Pedro Sato'. Goiabas tratadas com 1-MCP apresentam maior período de conservação em função do atraso no amadurecimento (Basseto et al., 2005; Linhares et al., 2007). O 1-MCP é um composto volátil que tem demonstrado ser um potente inibidor da ação do etileno na célula. Este produto se liga preferencialmente ao sítio de ligação do etileno, inibindo seu estímulo fisiológico sobre - amadurecimento (Watkins, 2008). Menor incidência de goiabas com podridões, causadas principalmente por C. gloeosporioides, também foi constatada por Basseto et al. (2005) após tratamento com 1-MCP.

Recobrimentos comestíveis podem contribuir para aumentar o período de conservação das goiabas, a exemplo da fécula de mandioca que tem sido citada como matéria prima adequada por formar uma película transparente, proporcionando bom aspecto e brilho, tornando frutas e hortaliças comercialmente atrativas e, por não ser tóxica, pode ser ingerida com o produto. O biofilme de fécula de mandioca é facilmente removido com água e apresenta-se como um produto comercial de baixo custo. Resultados satisfatórios na conservação de goiabas de polpa 
branca 'Kumagai' com fécula de mandioca, através do atraso no amadurecimento e consequentemente no aparecimento de podridões foram observados por Cerqueira (2007).

A hidrotermia foi eficaz na redução de pinta preta quando em associação com a fécula de mandioca, embora não tenha proporcionado controle isoladamente. O controle das doenças com o tratamento hidrotérmico ocorre desde que os esporos dos patógenos e as infecções quiescentes estejam na superfície ou nas primeiras camadas celulares da epiderme do fruto e que os esporos não sejam termo-resistentes. Pode atuar também inibindo o amadurecimento, atrasando o colapso e a extinção de compostos antifúngicos pré-formados presentes nas frutas (Lurie \& Pedreschi, 2014). Segundo Palou et al. (2008), a hidrotermia pode induzir a síntese de compostos antifúngicos, como fitoalexinas ou proteínas relacionadas à patogênese. Atraso no amadurecimento e controle da antracnose da goiaba, com redução do diâmetro das lesões em frutos inoculados e no número de lesões naturais foram constatados por Ferraz (2010).

Assim como a hidrotermia, o etanol+cloro associado a fécula foi eficiente em reduzir a incidência da pinta preta, diferentemente de quando aplicado isoladamente. Em goiabas 'Kumagai' submetidas à imersão em etanol $50 \%$, durante dois minutos, Ponzo (2009) conseguiu reduzir a incidência e a severidade da antracnose em frutos inoculados com C. gloeosporioides, sem alterar os atributos físicoquímicos dos frutos. O etanol é muito utilizado no processo de desinfestação, ou seja, processo que elimina os micro-organismos, atuando na desnaturação das proteínas e remoção de lipídios da membrana plasmática ou parede celular do micro-organismo (Venturelli et al, 2009), colaborando também na quebra da tensão superficial do fruto e com isso, possivelmente, favorecendo a atuação do segundo desinfetante, no caso o cloro. O mecanismo de ação do cloro não é bem conhecido, embora algumas hipóteses sugerem que há uma combinação com proteínas da membrana celular dos micro-organismos, assim formando compostos tóxicos e levando à inibição das enzimas essenciais (Donini et al., 2005).

Analisando o possível efeito sinérgico dos tratamentos, apenas a associação etanol+cloro/ fécula de mandioca foi proporcionalmente superior que os tratamentos individualizados, comportando-se como a associação mais eficiente no controle da pinta preta ao final do armazenamento. Ferraz (2010) obteve controle da antracnose quando associou a hidrotermia seguida do tratamento com cloreto de cálcio em goiabas orgânicas, ou seguida de fosfito de potássio em frutos procedentes de sistema de cultivo convencional.

A ocorrência da pinta preta foi superior na segunda fase dos experimentos, relacionado possivelmente a elevação em $3^{\circ} \mathrm{C}$ da temperatura de armazenamento dos frutos, de 22 para $25^{\circ} \mathrm{C}$, considerando que os frutos foram provenientes do mesmo pomar, embora de safras subsequentes. Em estudo do monociclo de Guignardia psidii em goiabas a máxima germinação e formação de apressório de $G$. psidii foi observada entre 25 e $30^{\circ} \mathrm{C}$ (Escanferla et al., 2009).

A incidência de pinta preta foi crescente com o estádio de maturação das goiabas. Resultados semelhantes foram obtidos por Azzolini et al. (2004) em que goiabas 'Pedro Sato' no estádio de maturação 1 apresentaram período de comercialização de seis dias quando armazenadas a $25^{\circ} \mathrm{C}$, enquanto frutos nos estádios 2 e 3 tiveram este período reduzido para quatro e dois dias, respectivamente, devido, principalmente, à ocorrência de podridões. Estudando o processo infeccioso de G. psidii em goiaba, Escanferla et al. (2009) observaram um aumento gradual na germinação e formação de apressórios do patógeno com a idade do fruto, indicando que frutos mais velhos são mais propensos à infecção. Segundo os autores, isto parece estar relacionado com certas modificações na superfície de frutas durante o seu processo de envelhecimento, como perda de tricomas.

O armazenamento dos frutos por oito dias a $22-25^{\circ} \mathrm{C}$ visou à expressão da pinta preta presente nos frutos em sua totalidade, entretanto, se considerarmos que as podridões desqualificam a fruta para comercialização 
Fischer et al. (2016) / Incidência de pinta preta e características...

e não devem preferencialmente ultrapassar $20 \%$ durante a vida útil (Fischer et al., 2011), a viabilidade dos frutos no tratamento controle ficou entre dois e quatro dias, em função do estádio de maturação. Contudo, com a associação etanol+cloro/fécula conseguiu-se aumento de dois a quatro dias na viabilidade destes frutos em função do atraso no aparecimento dos sintomas de pinta preta.

A utilização de 1-MCP e de fécula de mandioca foi eficiente em manter a qualidade físico-química das goiabas, retardando a mudança de coloração da casca e contribuindo para a retenção da firmeza da polpa, e consequentemente, estando diretamente relacionado à inibição do aparecimento dos sintomas de pinta preta.

A perda de massa em frutos é oriunda da respiração e transpiração. A possível redução desta perda por meio da aplicação do 1-MCP está relacionada ao fato do mesmo reduzir a respiração. A respiração reduz a massa do fruto, mas em menor proporção que a transpiração. Isso pode explicar o fato do 1-MCP não ter sido eficaz em retardar a perda de massa. Bassetto et al. (2005) também não verificaram redução da perda de massa em goiabas 'Pedro Sato' tratadas com 1-MCP. Já os recobrimentos a base de polissacarídeos, como é o caso da fécula, possuem, sob certas condições de temperatura e umidade relativa uma boa barreira ao $\mathrm{O}_{2} \mathrm{e}$ $\mathrm{CO}_{2}$, mas uma fraca barreira ao vapor d'água (Garcia et al., 1998). Isso pode explicar o fato da fécula não ter sido eficaz em retardar a perda de massa. Cerqueira (2007) também observou pequena redução da perda de massa em goiabas 'Kumagai' armazenadas a $22^{\circ} \mathrm{C} e$ tratadas com fécula $2 \%$.

Durante $\bigcirc$ amadurecimento, o fitorregulador etileno presente nos espaços intercelulares liga-se ao seu receptor na célula e desencadeia uma série de eventos que culminam com o amadurecimento e senescência do fruto (Barry \& Giovannoni, 2007). Os principais processos envolvidos na perda da coloração verde dos frutos durante $\mathrm{o}$ amadurecimento são a degradação da clorofila e a síntese de caroteno (Khandaker et al., 2013). A atividade da clorofilase, assim como de outras enzimas relacionadas ao processo de amadurecimento dos frutos dependem do etileno. Azzolini et al. (2004), observaram que a cor da casca apresentou diminuição dos valores de ângulo ('h) em todos os estádios de maturação durante o armazenamento, indicando a mudança de cor verde para amarela.

Watkins (2008) relata que o 1-MCP temse mostrado bastante eficiente em reduzir a produção e bloquear a ação do etileno em diversas espécies de flores, frutos e hortaliças. Esse composto liga-se permanentemente ao sítio de ligação do etileno na célula, evitando, por certo tempo, a ação do mesmo sobre os processos fisiológicos do amadurecimento. A sensibilidade do tecido vegetal ao etileno, com consequente amadurecimento do fruto, retorna devido à síntese de novos sítios de ligação. Cerqueira et al. (2009) verificaram que quanto maior a concentração de 1-MCP, melhor seu desempenho na retenção da cor verde da casca em goiabas 'Kumagai'. Em relação à firmeza da polpa, Basseto et al. (2005) também verificaram que goiabas 'Pedro Sato' tratadas com $900 \mathrm{~nL} \mathrm{~L}^{-1}$ de 1-MCP por três horas, mantiveram a firmeza por oito dias. A eficácia dos tratamentos a base de fécula em atrasar o amadurecimento dos frutos, deve-se à barreira formada pelo biofilme aos gases, prociciando redução da respiração dos frutos (Garcia et al., 1998).

O teor de açúcares aumenta com - amadurecimento das frutas por meio de processos biossintéticos ou pela degradação de polissacarídeos. Esse aumento pode ser devido à conversão de polissacarídeos da parede celular em açúcares solúveis. No entanto, na senescência ocorre redução no teor de açúcar (Chitarra \& Chitarra, 2005). Ponzo (2009) relata que após a colheita o teor de sólidos solúveis em goiaba parece não sofrer alterações significativas, tal fato pode ser explicado pelo baixo teor de amido em goiabas. Azzolini et al. (2004), relataram poucas mudanças no teor de sólidos solúveis em função dos estádios de maturação, a exemplo do observado no presente estudo em que o teor de sólidos solúveis dos frutos diferenciaram em menos de $1^{\circ}$ Brix. A associação contendo 1-MCP destacou-se em manter ou mesmo proporcionar leve aumento 
no teor de açúcares. Em goiabas 'Safeda' armazenadas a temperatura ambiente o teor de sólidos solúveis também aumentou com o tratamento com 1-MCP, enquanto que frutos armazenados $a 10^{\circ} \mathrm{C}$ esse aumento foi restringido (Singh \& Pal, 2008).

A maior acidez no tratamento com 1-MCP pode ser explicada pela retenção do amadurecimento. Bassetto et al. (2005) relataram maior acidez em goiabas tratadas com 1-MCP e armazenadas em temperatura ambiente. A redução nos teores de acidez titulável com o avanço do estádio de maturação dos frutos é decorrente da metabolização de ácidos voláteis e não voláteis no processo de amadurecimento (Chitarra \& Chitarra, 2005).

O aumento do ratio com o avanço do estádio de maturação é explicado pela redução da acidez enquanto o menor ratio observado nos frutos dos estádios 2 e 3 tratados com 1-MCP e fécula decorre do retardo no processo de amadurecimento.

Observou-se correlação entre o estádio de maturação, expresso pela cor da casca e a ocorrência de pinta preta em goiabas, fato também constatado por Azzolini et al. (2004) para antracnose. Associações entre o aumento das infecções com mudanças em atributos físico-químicos foram relatadas em manga (Lechaudel \& Joas, 2007) e pêssego (Souza, 2007). Nos frutos submetidos as associações de tratamentos, a correlação entre a pinta preta e os parâmetros cor da casca e firmeza da polpa em goiabas na associação etanol+cloro/fécula evidencia que a maior redução da doença está diretamente relacionada ao atraso da maturação dos frutos. Segundo Cavalini et al. (2006), a cor da casca e a firmeza da polpa são os índices de maturação mais adequados para goiaba, permitindo diferenciar os estádios de maturação e apresentam boa correlação entre si.

\section{Conclusões}

A ocorrência da pinta preta foi crescente com o estádio de maturação dos frutos e a associação de tratamento etanol+cloro/ fécula de mandioca destacou-se em reduzir a incidência de doença em goiabas 'Pedro
Sato', correlacionado ao atraso da maturação, mantendo a viabilidade dos frutos por até oito dias nos estádios 1 e 2 de maturação.

A manutenção da qualidade físicoquímica das goiabas 'Pedro Sato' com as associações de tratamentos foram evidenciadas pelo atraso na mudança de coloração da casca e menor redução na firmeza.

\section{Agradecimento}

À FAPESP (Proc. 2012/07207-7) pelo apoio financeiro concedido ao projeto.

\section{Referências}

Azzolini, M., Jacomino, A.P., Bron, I.U. 2004. Índices para avaliar qualidade pós-colheita de goiabas em diferentes estádios de maturação. Pesquisa Agropecuária Brasileira 39: 139-145.

Barry, C.B., Giovannoni, J.J. 2007. Ethylene and fruit ripening. Journal of Plant Growth Regulation 26: 143-159.

Bassetto, E., Jacomino, A.P., Pinheiro, A.L. 2005. Conservation of 'Pedro Sato' guavas under treatment with 1-methylcyclopropene. Pesquisa Agropecuária Brasileira 40: 433-440.

Cavalini, F.C., Jacomino, A.P., Lochoski, M.A., Kluge, R.A., Ortega, E.M.M. 2006. Maturity indexes for Kumagai and Paluma guavas. Revista Brasileira de Fruticultura 28: 176-179.

Cerqueira, T.S. 2007. Recobrimentos comestíveis em goiabas cv. Kumagai. 69f. (Dissertação de Mestrado) - Escola Superior de Agricultura "Luiz de Queiroz", Universidade de São Paulo, Piracicaba, Brasil.

Cerqueira, T.S., Jacomino, A.P.,Sasaki, F.F., Amorim, L. 2009. Controle do amadurecimento de goiabas 'Kumagai' tratados com 1-Metilciclopropeno. Revista Brasileira de Fruticultura 31: 687-692.

Chitarra, M.I.F., Chitarra, A.B. 2005. Pós-colheita de frutas e hortaliças - fisiologia e manuseio. $2^{a}$ ed. EDUFLA, Lavras, Brasil, 783 p.

Donini, L.P., Souza, J.A., Moura, I.F., Guisso, A.P., Viégas, J. 2005. Preparo de lâminas foliares de Aráceas ornamentais: desinfestação com diferentes concentrações de hipoclorito de sódio. Arquivos do Instituto Biológico 72: 517-522.

Escanferla, M.E., Moraes, S.R.G., Salaroli, R.B., Massila JR. 2009. Pre-penetration stages of Guignardia psidii in guava: effect of temperature, wetness duration and fruit age. Journal of Phytopathology 157: 618-624.

Ferraz, D.M.M. 2010. Controle da antracnose 
(Colletotrichum gloeosporioides) em póscolheita da goiaba (Psidium guajava), produzida em sistema de cultivo convencional e orgânico, pela aplicação de fosfitos, hidrotermia e cloreto de cálcio. 119f. (Dissertação de Mestrado) Universidade de Brasília, Brasília, Brasil.

Fischer, I.H., Almeida, A.M., Arruda, M.C., Bertani, M.A.R., Garcia, M.J.M., Amorim, L. 2011. Danos em pós-colheita de goiabas na Região do Centro-Oeste Paulista. Bragantia 70: 570-576.

Fischer, I.H., Silva, B.L., Soares, A.R., Arruda, M.C., Parisi, M.C.M., Amorim, L. 2012. Efeito de fungicidas e produtos alternativos no controle da antracnose e da pinta preta da goiaba. Semina: Ciências Agrárias 33: 2753-2766.

Garcia, M.A., Martino, M.N., Zaritzky, N.E. 1998. Plasticized starch-based coatings to improve strawberry (Fragaria $x$ Ananassa) quality and stability. Journal of Agricultural and Food Chemistry 46: 3578-3767.

Hojo, R.H., Chalfun, N.N.J., Hojo, E.T.D.; Veiga, R.D., Paglis, C.M., Lima, L.C.O. 2007. Produção e qualidade dos frutos da goiabeira 'Pedro Sato' submetida a diferentes épocas de poda. Pesquisa Agropecuária Brasileira 42: 357362.

Khandaker, M.M., Boyce, A.N., Osman, N., Golam, F., Rahman, M.M., Sofian-Azirun, M. 2013. Fruit development, pigmentation and biochemical properties of wax apple as affected by localized application of $\mathrm{GA}_{3}$ under field conditions. Brazilian Archives of Biology and Technology 56: 11-20.

Léchaudel, M., Joas, J. 2007. An overview of preharvest factors influencing mango fruit growth, quality and postharvest behaviour. Brazilian Journal of Plant Physiology 19: 287-298.

Linhares, L.A., Santos, C.D., Abreu, C.M.P., Corrêa, A.D. 2007. Transformações químicas, físicas e enzimáticas de goiabas Pedro Sato tratadas na pós-colheita com cloreto de cálcio e 1-metilciclopropeno e armazenadas sob refrigeração. Ciência e Agrotecnologia 31: 829841.

Lurie, S., Pedreschi, R. 2014. Fundamental aspects of postharvest heat treatments. Horticulture Research 1:1-7.

Martins, M.C., Amorim, L., Lourenço, S.A., Gutierrez, A.S.D., Watanabe, H.S. 2007. Incidência de danos pós-colheita em goiabas no mercado atacadista de São Paulo e sua relação com a prática de ensacamento dos frutos. Revista Brasileira de Fruticultura 29: 245-248.

Palou, L., Smilanick, J.L., Droby, S. 2008. Alternatives to conventional fungicides for the control of citrus postharvest green and blue moulds. Stewart Postharvest Review 2: 1-17.

Ponzo, F.S. 2009. Agentes alternativos no controle pós-colheita da antracnose em goiabas 'Kumagai'. 77f. (Dissertação de Mestrado) Instituto Agronômico, Campinas, Campinas, Brasil.

Risterucci, A.M., Duval, I. M.F., Rohde, W., Billotte, N. 2005. Isolation and characterization of microsatellite loci from Psidium guajava L. Molecular Ecology Notes 5: 745-748.

Singh, S.P., Pal, R.R. 2008. Response of climacterictype guava (Psidium guajava L.) to postharvest treatment with 1-MCP. Postharvest Biology and Technology 47: 307-314.

Soares-Colletti, A.R. Fischer, I.H., Lourenço, S.A. 2014. The incidence of postharvest diseases on 'Kumagai' and 'Pedro Sato' guavas at wholesale markets in Brazil. Tropical Plant Pathology 39: 478482.

Souza, D.C. 2007. Progresso temporal e padrão espacial de epidemias da podridão parda do pessegueiro. 63f. (Dissertação de Mestrado) Escola Superior de Agricultura Luiz de Queiroz, Universidade de São Paulo, Piracicaba, Brasil.

Venturelli, A.C., Torres, F.C., Almeida-Pedrin, R.R., Almeida, R.R., Almeida, M.R., Ferreira, F.P.C. 2009. Avaliação microbiológica da contaminação residual em diferentes tipos de alicates ortodônticos após desinfecção com álcool $70 \%$. Revista Dental Press de Ortodontia e Ortopedia Facial 14: 43-52.

Vila, M.T.R., Lima, L.C.O., Boas, E.V.B.V., Hojo, E.T.D., Rodrigues, L.J., Paula, N.R.F. 2007. Chemical and biochemical characterization of guavas stored under refrigeration and modified atmosphere. Ciência e Agrotecnologia 31: 1435-1442.

Watkins, C.B. 2008. Overview of 1-Methylcyclopropene trials and uses for edible horticultural crops. Hort-Science 43: 86-94.

Zar, J.H. 1999. Biostatistical analyis. Prentice-Hall, New Jersey. USA. 663 p. 